\title{
Skeletal muscle of gastric cancer patients expresses genes involved in muscle regeneration
}

\author{
PATRIZIA PESSINA ${ }^{1,2}$, VALENTINA CONTI $^{2}$, FABIO PACELLI $^{3}$, FAUSTO ROSA $^{3}$, \\ GIOVAN BATTISTA DOGLIETTO ${ }^{3}$, SILVIA BRUNELLI ${ }^{1,2}$ and MAURIZIO BOSSOLA ${ }^{3}$
}

\author{
${ }^{1}$ Department of Experimental Medicine, University of Milano-Bicocca, 20052 Monza ; ${ }^{2}$ Division of Regenerative Medicine, \\ H. San Raffaele Scientific Institute, 20132 Milan; ${ }^{3}$ Department of Surgery, Catholic University, 00168 Rome, Italy
}

Received April 23, 2010; Accepted May 26, 2010

DOI: 10.3892/or_00000916

\begin{abstract}
Experimental studies have suggested that defective skeletal muscle regeneration could contribute to muscle wasting in cancer patients. However, data in humans are still lacking. In this study we aimed to assess the expression of the genes involved in muscle regeneration in gastric cancer patients. The RNA expression of the genes involved in muscle regeneration was assessed in the rectus abdominis muscle of patients with gastric cancer $(n=30)$ and in age-matched control subjects $(\mathrm{n}=8)$. The Pax7 expression was significantly increased in the muscle of gastric cancer patients, either in the first stages of the disease or in stages IIIA and B. The increased expression was present both in stages IA and B and in stages II and III. The MyoD espression was also higher in the cancer patients than in the controls. However, the increased MyoD expression was present only in stages IA and B and not in the more advanced stages of the disease. The Myf5 expression, as well as that of the neonatal isoform of Myosin Heavy Chain (nMHC) did not differ significantly between the cancer patients and the controls. The necdin expression was negligible in healthy adult muscles and was significantly up-regulated in the muscle of gastric cancer patients. Its expression was highly increased in stages IA and B while it was similar to the control in stages II and III. The results of the present study show that in the skeletal muscle of gastric cancer patients, the expression of the genes involved in muscle regeneration is increased with respect to the controls.
\end{abstract}

\section{Introduction}

Cancer cachexia is a debilitating and life-threatening syndrome characterised by anorexia, body weight loss, loss of adipose

Correspondence to: Dr Maurizio Bossola, Department of Surgery, Catholic University, 00168 Rome, Italy

E-mail:maubosso@tin.it

Dr Silvia Brunelli, H. San Raffaele Scientific Institute, Via Olgettina 60, 20132 Milano, Italy

E-mail: brunelli.silvia@hsr.it

Key words: skeletal muscle, wasting, cancer, regeneration, necdin tissue and skeletal muscle (1). Cachexia accounts for at least $20 \%$ of deaths in neoplastic patients. Cancer cachexia significantly impairs the quality of life as well as the response to anti-neoplastic therapies, thus increasing the morbidity and mortality of cancer patients. Muscle wasting is the most important phenotypic feature of cancer cachexia and the principal cause of function impairment, fatigue and respiratory complications, mainly related to the hyperactivation of muscle proteolytic pathways. The loss of muscle protein, defined as muscle catabolism, occurs due to an imbalance between the rates of muscle protein synthesis and breakdown (1). In recent years, numerous studies have contributed to our understanding of the mechanisms underlying the increase of skeletal muscle protein breakdown in cancer cachexia and to the clarification of the relative contribution of different proteolytic pathways $(2,3)$. Four major proteolytic systems exist in the skeletal muscle: The endosome-lysosome system that relies on the activity of acidic proteases, the caspases, the calpains, and the ubiquitinproteasome system $(2,3)$. The bulk of muscle proteins (50$70 \%$ ) exists in the actomyosin complexes and myofibrils, and in different catabolic conditions, contractile proteins in these complexes are degraded by the ubiquitin-proteasome system (4). However, the ubiqutin-proteasome system degrades monomeric actin or myosin but does not degrade the actomyosin complexes (4). Moreover, the initial steps involved in myofibrillar proteolysis are essentially unknown, two systems being the major candidates for this role, the caspase- 3 and the calpains (1).

Another factor contributing to the loss of muscle mass could be a decrease in skeletal muscle regeneration. Skeletal muscle tissue upon certain physiological stimuli or in pathological conditions, is able to undergo an extensive repair process aimed at preventing the loss of muscle mass (5). The key cells in this process are satellite cells (6). During the perinatal phases of muscle growth and upon any muscle injury, satellite cells are activated, and they start proliferating and expressing Myf5 and MyoD, while the Pax7 expression is progressively reduced. At this stage they are often referred to as myogenic precursor cells (5). Subsequently, the expression of myogenin and MRF4 (MRF members) is up-regulated in the cells beginning their terminal differentiation program, followed by a permanent exit from the cell cycle, the activation of muscle specific proteins, such as sarcomeric myosin, 
and fusion with damaged muscle fibers or with themselves to produce new fibers to replace the dead ones.

Defective skeletal muscle regeneration secondary to the potent inhibiting action of TNF- $\alpha$ has been shown to contribute to muscle wasting in a mouse model of cachexia $(7,8)$. Indeed, we have demonstrated that necdin, a member of the MAGE family, plays an important role in skeletal muscle growth and repair in vivo, and is selectively expressed in the muscles of cachetic mice and that its expression is causally linked to a protective response of the tissue against tumor-induced wasting, the inhibition of myogenic differentiation and fiber regeneration (9). Despite such experimental evidence, studies on the role of muscle regeneration or defective regeneration in humans with cancer are still lacking. The present study aimed at evaluating the expression of the genes involved in muscle regeneration in the muscle of gastric cancer patients and age-matched controls. We show that the muscles of gastric cancer patients express higher levels of Pax7, MyoD and necdin, suggesting that the regeneration process is not inhibited, but rather is activated, possibly to physiologically counteract tumor-induced muscle wasting.

\section{Patients and methods}

Subjects. This study was approved by the local ethics committees. Thirty consecutive patients with gastric cancer admitted to the Istituto di Clinica Chirurgica of the Università Cattolica del Sacro Cuore of Rome, Italy, between January 2009 and September 2009, were included in the study. The diagnosis of gastric cancer was made by an endoscopic biopsy. Eight patients undergoing surgery for benign abdominal diseases (laparocele, 6 cases; inguinal hernia, 2 cases) served as the control group. Exclusion criteria for both groups were as follows: Acute or chronic renal failure (serum creatinine $>1.2 \mathrm{mg} / \mathrm{dl}$ ), liver failure, diabetes, metabolic acidosis, sepsis, AIDS, inflammatory bowel disease, auto-immune disorders, chronic heart failure, acute and chronic hepatitis, hyperthyroidism, and chronic obstructive pulmonary disease.

Protocol. Written informed consent for the study procedures was obtained from the patients. All the subjects were studied at 8 a.m., after overnight fasting. Blood samples for subsequent biochemical analyses were obtained from an antecubital vein immediately before entering the operating room.

Nutritional assessment. The nutritional assessment included anthropometric (actual body weight, usual body weight and percentage weight loss) and biochemical (serum albumin) indices.

Muscle biopsy. A biopsy specimen was obtained from the rectus abdominis muscle during the initial phase of the operation. After skin incision and dissection through the subcutaneous fat, the anterior sheet of the rectus abdominis muscle was opened with scissors and a muscle biopsy specimen weighing $0.5 \mathrm{~g}$ was obtained. The biopsy specimen was immediately frozen in liquid nitrogen and then stored at $-70^{\circ} \mathrm{C}$ until analysis. After the muscle biopsy had been obtained, small bleeding vessels were carefully controlled with ligatures and cautery, whereafter the operation continued in a
Table I. Characteristics of patients.

\begin{tabular}{lccr}
\hline & Controls & $\begin{array}{c}\text { Gastric cancer } \\
\text { patients } \\
(\mathrm{n}=30)\end{array}$ & P-value \\
& $(\mathrm{n}=8)$ & $63.8 \pm 2.8$ & 0.45 \\
Age (years) & $64.2 \pm 2.6$ & $17: 13$ & 0.92 \\
Gender (M:F) & $5: 3$ & $10.1 \pm 3.1$ & $<0.001$ \\
Weight loss $(\%)^{*}$ & 0 & $3.3 \pm 0.4$ & $<0.001$ \\
Serum albumin $(\mathrm{g} / \mathrm{dl})^{*}$ & $4.2 \pm 0.1$ & & \\
Stage & & 17 & \\
IA and B & & 4 & \\
II & & 9 & \\
IIIA and B & & & \\
\hline
\end{tabular}

Data are expressed as mean $\pm \mathrm{SD}$. Stage of disease is according to the UICC classification of tumor stage (16).

routine fashion. No complications occurred from the biopsy procedure.

RNA isolation and real-time PCR analysis. Total RNA (1 $\mu \mathrm{g})$ isolated using the TRIzol protocol (Invitrogen) was converted into double-stranded cDNA using the cDNA synthesis High Capacity Reverse Transcription Kit (Applied Biosystems), according to the manufacturer's instructions. Real-time quantitative PCR was carried out with a real-time PCR system (Mx3000P; Stratagene). Each cDNA sample was amplified in duplicate using the SYBR-Green Supermix (Bio-Rad Laboratories) and using the following specific primers: GAPDH forward, 5' TTCACCACCATGGAGAAG GC 3' and reverse, 5' GGCATGGACTGTGGTCATGA 3'; Myf5 forward, 5' TGAGAGAGCAGGTGGAG AACTAC 3' and reverse, 5' AGGTGATCCGGTCCA CTATG 3'; MyoD forward, 5' AGCACTACAGCGGCGACT 3' and reverse, 5' GCGACTCAGAAGGCACGTC 3'; necdin forward, 5' GA GTTTGCCCTGGTCAAAGC 3' and reverse, 5' CATGG GCATACGGTTGTTGAG 3'; nMHC forward, 5' CACT TGGAGTTTATCCACCAGATCC 3' and reverse, 5' TGA AGAAGGAGCAGGACACCAG 3'; Pax7 forward, 5' CAC TGTGACCGAAGCACTGT 3' and reverse, 5' GTCAGGTT CCGACTCCACAT 3'.

Statistical analysis. A non-parametric t-statistic for unequal $\mathrm{n}$ was used to determine the statistical differences between the controls and cancer patient groups for the measurement of RNA expression $(\mathrm{P}<0.05)$. For bar graphs, the values plotted are mean \pm SEM.

\section{Results}

Patients' characteristics. As shown in Table I, the gastric cancer patients and the controls were similar in terms of age and sex distribution. Nevertheless, the gastric cancer patients showed a significantly higher percentage of weight loss and lower serum albumin levels.

Expression of PAX7 and MRF genes. Healthy adult muscle contains a small number of quiescent satellite cells and muscle 

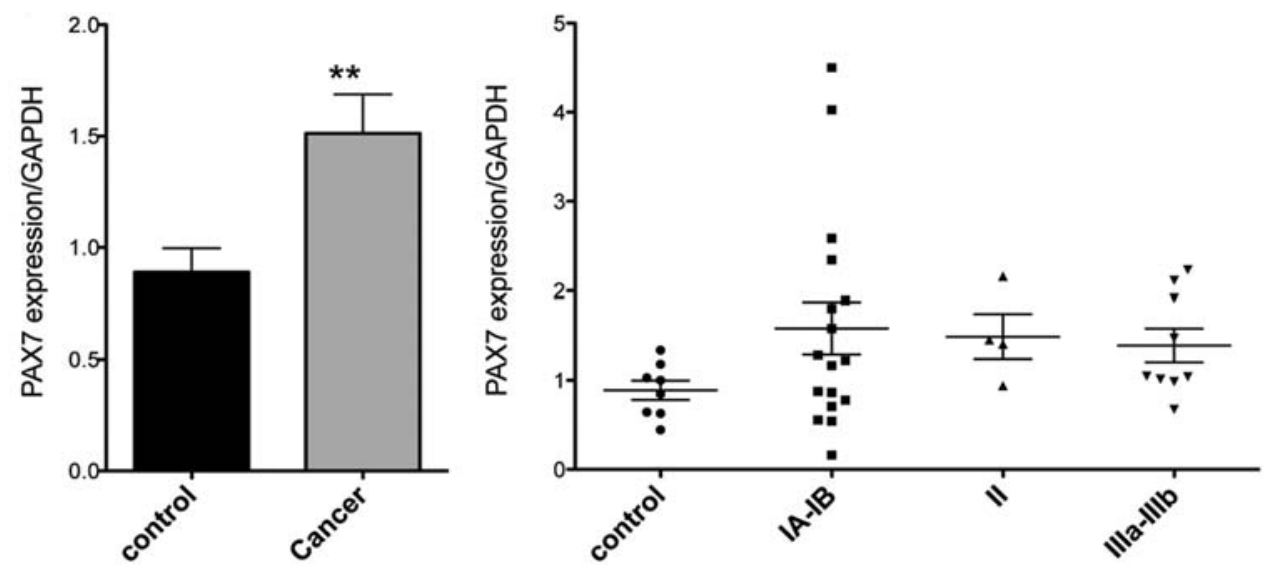

Figure 1. RNA expression of Pax7 in the skeletal muscle of the controls and gastric cancer patients (left panel, all cases; right panel, patients stratified according to the stage). ${ }^{* *}$ With respect to the controls, $\mathrm{P}<0.005$.
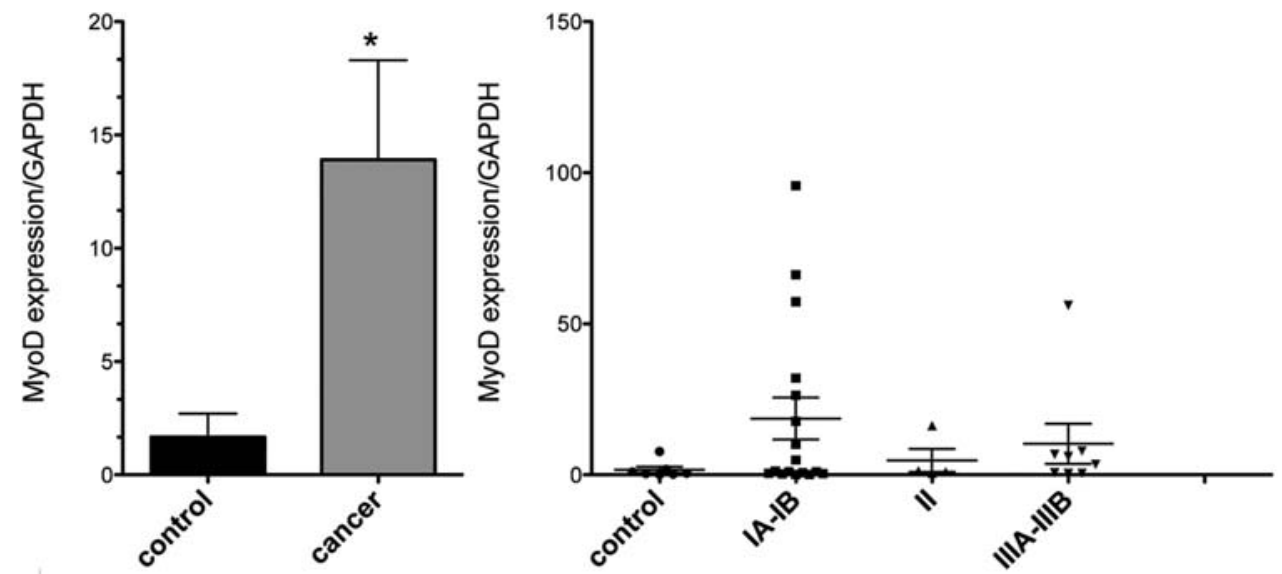

Figure 2. RNA expression of MyoD in the skeletal muscle of the controls and gastric cancer patients. (left panel, all cases; right panel, patients stratified according to the stage). "With respect to the controls, $\mathrm{P}<0.05$.
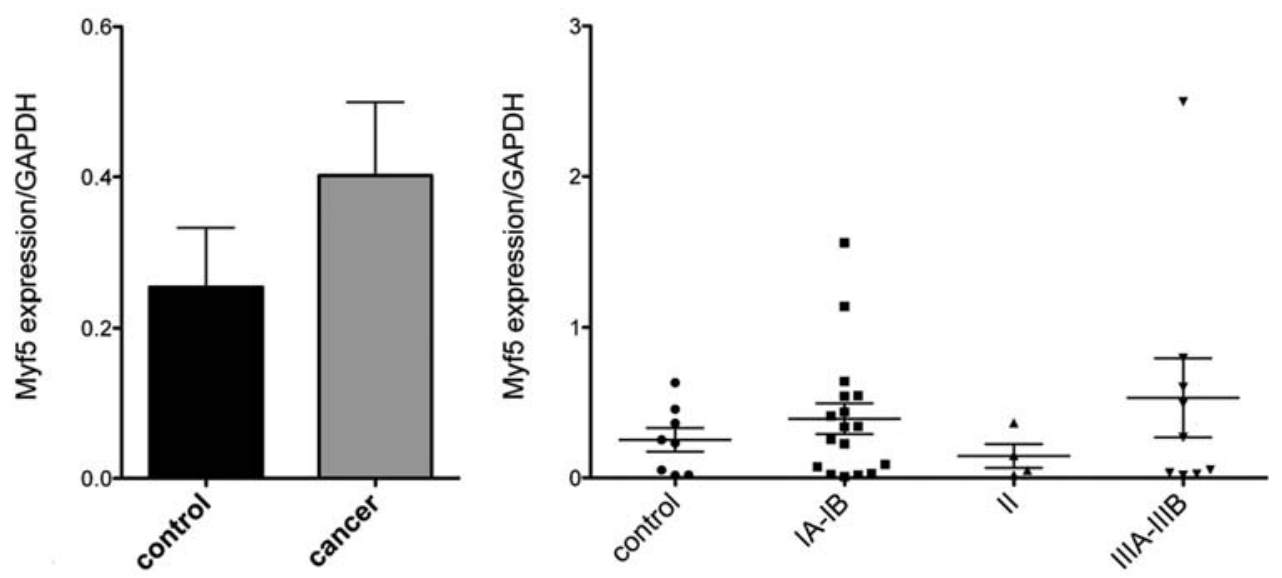

Figure 3. RNA expression of Myf5 in the skeletal muscle of the controls and gastric cancer patients (left panel, all cases; right panel, patients stratified according to the stage). Differences are not statistically significant.

precursors expressing Pax7. The Pax7 expression was significantly higher in the muscle of gastric cancer patients than in the controls (Fig. 1). The increased expression was present both in stages IA and B and in stages II and III. The MyoD espression was also higher in the cancer patients than in the controls (Fig. 2). However, the increased MyoD expression was present only in stages IA and B and not in the more advanced stages of the disease (Fig. 2). The Myf5 expression 

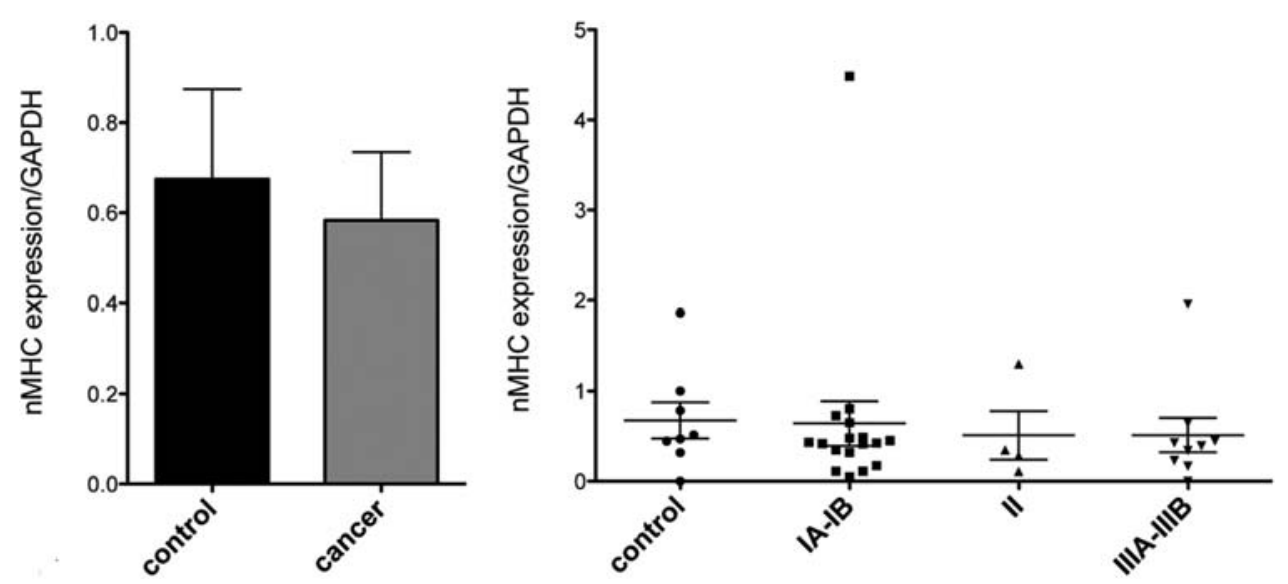

Figure 4. RNA expression of nMHC in the skeletal muscle of the controls and gastric cancer patients (left panel, all cases; right panel, patients stratified according to the stage). Differences are not statistically significant.
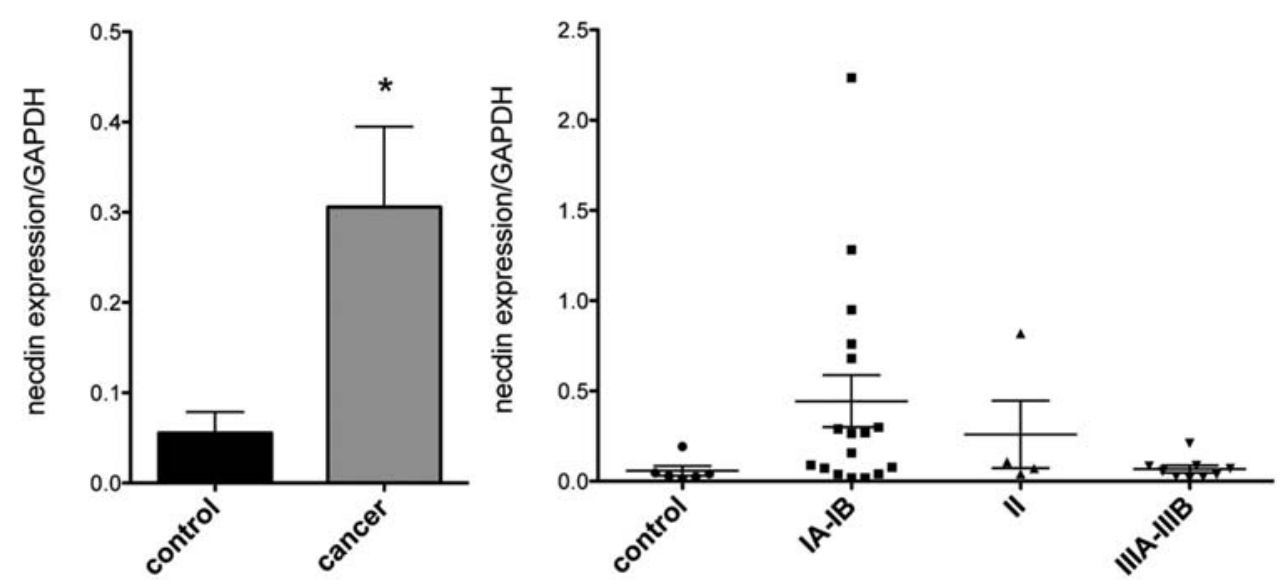

Figure 5. RNA expression of necdin in the skeletal muscle of the controls and gastric cancer patients (left panel, all cases; right panel, patients stratified according to the stage). ${ }^{*}$ With respect to the controls, $\mathrm{P}<0.01$; stage IA and B with respect to the controls, $\mathrm{P}<0.015$.

(Fig. 3), as well as that of the neonatal isoform of Myosin Heavy Chain (nMHC) did not differ significantly between cancer patients and controls (Fig. 4).

Expression of necdin. The necdin expression was negligible in the healthy adult muscles and was significantly upregulated in the muscle of gastric cancer patients (Fig. 5). Its expression was highly increased in stages IA and B while it was similar to the control in stages II and III (Fig. 5).

\section{Discussion}

The present study showed that in the skeletal muscle of cancer patients, the expression of the genes involved in muscle regeneration was increased with respect to the controls. To our knowledge, this is the first time that such results are reported, suggesting that in response to cancer and muscle wasting signals in the muscle possibly activate a regenerative response to try to counteract muscle hypercatabolism.

We studied the skeletal muscle of patients affected by gastric cancer as it is a well known model of muscle wasting. In fact, we have shown that in this model, the increased muscle proteasome activity correlates with disease severity (4) as well as the increased expression of ubiquitin mRNA (10).

Pax7 is un unbiased marker of satellite cells, both in their quiescent state as well as in the first phases of activation when they start to proliferate and express MyoD and Myf5 (11). Activated satellite cells can go back to quiescence, to maintain the myogenic stem cell pool, or proceed toward the final differentiation steps (12). The gastric cancer patients showed an increased muscle expression of Pax7 and MyoD. These data suggest that regeneration has been triggered. Pax7, but not MyoD, continues to be up-regulated in patients during the later stages of the disease, indicating that the number of satellite cells in these muscles is higher than in the controls. However, it is possible that these cells are no longer able to proceed toward terminal differentiation and formation of new fibers, as suggested by the lack of expression of nMHC, a marker of new regenerating fibers.

In a mouse model of cachexia myogenesis is affected at several levels. Studies have mainly focused on the endpoint of atrophy, the increased catabolism rate leading to the increased ubiquitin-dependent proteolysis of muscle specific structural proteins. This process is mainly linked to the 
activation of both IKKß/NF-кB-dependent and -independent pathways triggered by the increased signaling of TNF- $\alpha$ (13). $\mathrm{TNF}-\alpha$ has been also shown to inhibit in vitro and in vivo myogenesis $(7,9)$ as well as muscle regeneration by inhibiting cell fusion and by up-regulating caspase activity in myogenic stem cells (8). We have previously shown that the MAGE protein necdin is selectively expressed in the muscles of cachectic mice and have proven that its expression is causally linked to a protective response of the tissue against the tumor-induced wasting of myogenic differentiation and the inhibition of fiber regeneration (9). Necdin carries out this role mainly via interference with $\mathrm{TNF}-\alpha$ signaling at various levels, including the regulation of the expression of TNFR1 and the regulation of the activity of caspases. In this study we discovered that, similarly to that in the mouse model, the skeletal muscle of stage IA and B gastric cancer patients shows a mild but significant increase of necdin expression, suggesting an analogous response of the human muscle to the tumor load. The absence of a similar up-regulation of necdin during the later stages of the disease, could indicate that, as in the case of MyoD, the myoblasts still present in the affected muscle are impaired.

A delicate balance between cell proliferation and exit from the cell cycle, differentiation and fusion is required for the correct muscle regeneration to occur, in particular when this process becomes chronic as in the case of cancer-induced muscle wasting. This process could be deteriorated if the tissue is less efficient in counteracting the loss of muscle.

Most strategies for the prevention and treatment of cancer cachexia have proven to be only partially effective (14). The pharmacological inhibition of muscle protein catabolism has yielded some encouraging results, nevertheless clinical data are still contradictory. The stimulation of muscle anabolism could represent another promising therapeutic alternative. Recently, the attention has been focused on the use of insulinlike growth factor-1 (IGF-1) (15). IGF-1 plays a critical role in muscle homeostasis and its overexpression at the muscular level by gene therapy has been shown to reverse muscle hypotrophy secondary to catabolic conditions and to induce muscle hypertrophy by increasing muscle mass and strength.

Based on our findings, the process of regeneration could be another important therapeutic target. Improved regeneration, while maintaining the stem cells pool, is one of the current strategies for counteracting muscle loss in muscle degenerative diseases, including genetic diseases such as muscular dystrophies. It has been shown that the muscle- specific overexpression of necdin in a mouse model of cachexia not only has a high degree of efficacy in preventing tumor-induced muscle wasting, by improving regeneration, but also exerts beneficial effects on other tissues affected by the disease (9). Possibly, necdin could be considered as a candidate molecule for the design of new approaches for the prevention and treatment of cachexia in cancer patients.

\section{References}

1. Tisdale MJ: Mechanisms of cancer cachexia. Physiol Rev 89: 381-410, 2009.

2. Bossola M, Pacelli F, Tortorelli A and Doglietto GB: Cancer cachexia: it's time for more clinical trials. Ann Surg Oncol 14: 276-285, 2007.

3. Bossola M, Pacelli F and Doglietto GB: Novel treatments for cancer cachexia. Expert Opin Investig Drugs 16: 1241-1253, 2007.

4. Bossola M, Muscaritoli M, Costelli P, et al: Increased muscle proteasome activity correlates with disease severity in gastric cancer patients. Ann Surg 237: 384-389, 2003.

5. Zammit PS, Golding JP, Nagata Y, et al: Muscle satellite cells adopt divergent fates: a mechanism for self-renewal? J Cell Biol 166: 347-357, 2004.

6. Cossu G and Biressi S: Satellite cells, myoblasts and other occasional myogenic progenitors: possible origin, phenotypic features and role in muscle regeneration. Semin Cell Dev Biol 16: 623-631, 2005.

7. Oletti D, Moresi V, Adamo S, et al: Tumor necrosis factor-alpha gene transfer induces cachexia and inhibits muscle regeneration. Genesis 43: 120-128, 2005.

8. Moresi V, Pristerà A, Scicchitano $\mathrm{B}$, et al: TNF inhibition of skeletal muscle regeneration is mediated by a caspase dependent stem cell response. Stem Cells 26: 997-1008, 2008.

9. Sciorati C, Touvier T, Buono R, et al: Necdin is expressed in cachectic skeletal muscle to protect fibers from tumor-induced wasting. J Cell Sci 122: 1119-1125, 2009.

10. Bossola M, Muscaritoli M, Costelli P, et al: Increased muscle ubiquitin mRNA levels in gastric cancer patients. Am J Physiol Regul Integr Comp Physiol 280: R1518-R1523, 2001.

11. Zammit PS: All muscle satellite cells are equal, but are some more equal than others? J Cell Sci 121: 2975-2982, 2008.

12. Gayraud-Morel B, Chretien F and Tajbakhsh S: Skeletal muscle as a paradigm for regenerative biology and medicine. Regen Med 4: 293-319, 2009.

13. Cai D, Frantz JD, Tawa NE, et al: IKKbeta/NF-kappaB activation causes severe muscle wasting in mice. Cell 119: 285-298, 2004.

14. Murphy KT and Lynch GS: Update on emerging drugs for cancer cachexia. Expert Opin Emerg Drugs 14: 619-632, 2009.

15. Muscaritoli M, Bossola M, Aversa Z, et al: Prevention and treatment of cancer cachexia: New insights into an old problem. Eur J Cancer 42: 31-41, 2006.

16. Union International Contre le Cancer: TNM Classification of Malignant Tumors. 4th edition, Geneve, 1987. 